\title{
Towards the improved quantification of in vivo abnormal wall shear stresses in BAV-affected patients from 4D-flow imaging: Benchmarking and application to real data
}

\author{
F. Piatti ${ }^{a}$, S. Pirola ${ }^{\text {b }}$, M. Bissell ${ }^{\text {c }}$, I. Nesteruk ${ }^{\text {d }}$, F. Sturla ${ }^{a}$, A. Della Corte ${ }^{\text {e }}$, A. Redaelli $^{a}$, E. Votta $^{\text {a,* }}$ \\ ${ }^{a}$ Department of Electronics, Information and Bioengineering, Politecnico di Milano, Milano, Italy \\ ${ }^{\mathrm{b}}$ Department of Chemical Engineering, Imperial College, London, United Kingdom \\ ${ }^{\mathrm{c}}$ Division of Cardiovascular Medicine, Radcliffe Department of Medicine, University of Oxford, Oxford, United Kingdom \\ ${ }^{\mathrm{d}}$ Institute of Hydromechanics, National Academy of Sciences of Ukraine, Kyiv, Ukraine \\ e Department of cardiothoracic and Respiratory Sciences, Second University of Naples, Naples, Italy
}

\section{ABSTRACT}

Bicuspid aortic valve (BAV), i.e. the fusion of two aortic valve cusps, is the most frequent congenital cardiac malformation. Its progression is often characterized by accelerated leaflet calcification and aortic wall dilation. These processes are likely enhanced by altered biomechanical stimuli, including fluid-dynamic wall shear stresses (WSS) acting on both the aortic wall and the aortic valve. Several studies have proposed the exploitation of 4D-flow magnetic resonance imaging sequences to characterize abnormal in vivo WSS in BAV-affected patients, to support prognosis and timing of intervention. How-ever, current methods fail to quantify WSS peak values.

On this basis, we developed two new methods for the improved quantification of in vivo WSS acting on the aortic wall based on 4D-flow data.

We tested both methods separately and in combination on synthetic datasets obtained by two computational fluid-dynamics (CFD) models of the aorta with healthy and bicuspid aortic valve. Tests highlighted the need for data spatial resolution at least comparable to current clinical guidelines, the low sensitivity of the methods to data noise, and their capability, when used jointly, to compute more rea-listic peak WSS values as compared to state-ofthe-art methods.

The integrated application of the two methods on the real 4D-flow data from a preliminary cohort of three healthy volunteers and three BAV-affected patients confirmed these indications. In particular, quantified WSS peak values were one order of magnitude higher than those reported in previous $4 \mathrm{D}-$ flow studies, and much closer to those computed by highly time- and space-resolved CFD simulations.

\section{Introduction}

Bicuspid aortic valve (BAV) consists in the congenital fusion of two of the three aortic valve (AV) leaflets. BAV is the most common congenital cardiac disease and affects about $2 \%$ of newborns (Michelena et al., 2014). BAV is a recognized risk factor for calcific aortic valve stenosis and for ascending aorta dilation: approximately one half of the patients undergoing surgery to treat calcific aortic stenosis are affected by BAV (Roberts and Ko, 2005), and more than $50 \%$ of BAV patients develop ascending aorta aneurysm (Fedak

\footnotetext{
* Correspondence to: Department of Electronics, Information and Bioengineer-

ing, Politecnico di Milano Via Golgi 39, 20133 Milano, Italy.

E-mail address: emiliano.votta@polimi.it (E. Votta).
}

et al., 2002; Nistri et al., 1999). Alterations in aortic root structural mechanics (Conti et al., 2010a, 2010b) and fluid-dynamics (Hope et al., 2014) induced by BAV abnormal morphology have been identified as key factors determining or accelerating these degenerative processes. These alterations include abnormal fluiddynamic wall shear stresses (WSS) acting on the endothelial cells covering the inner surface of the aortic wall and the aortic valve leaflets, respectively. Although through mechanisms that are not fully understood, altered WSS can trigger mechanotransduction path-ways leading to altered gene and protein expression, to inflammatory processes, and to altered regulation of interstitial cell pheno-types (Butcher and Nerem, 2006; El-Hamamsy et al., 2010). Currently, BAV risk stratification, prognosis and decision for surgery largely rely on purely geometrical criteria, as the measurement of the aortic diameter (Della Corte et al., 2014) and of the tilting angle 
characterizing the fused leaflets (Della Corte et al., 2012). These criteria are empirical and sub-optimal; biomechanically-driven ones could complement them allowing for more reliable prog-nosis and aid the decision process.

In this scenario, phase-contrast magnetic resonance imaging (PC-MRI), and in particular its latest evolution, i.e. 4D flow, has been proposed as a tool to quantify in vivo fluid-dynamic alterations affecting BAV patients (Bissell et al., 2013; Hope et al., 2014). $4 \mathrm{D}$-flow sequences offer the ability to capture complex 3D timeresolved velocity patterns, without any restriction to predefined $2 \mathrm{D}$ imaging planes (Markl et al., 2012), allowing for retrospective investigations of any location of the acquired volume. 4D-flow sequences' value is yet still hampered by low spatial-temporal resolution, long-lasting acquisitions and lack of standardized protocols (Markl et al., 2014). However, published studies have clearly shown that some hemodynamic markers (e.g. peak velocity, flow jet angle, helicity) can be reliably quantified to assess alterations in BAV (Lorenz et al., 2014; Sigovan et al., 2011; Stalder et al., 2008). By contrast, the reliability of WSS patterns estimations is still debated: differences in spatial trends of WSS between healthy subjects and BAV patients can be captured (Bissell et al., 2013), even though the corresponding numerical results are not valuable. In fact, there are evidences that underestimations in WSS values can occur primarily due to spatial resolution and noise issues of the acquired datasets (Petersson et al., 2012). Hence, currently the gross fluid-dynamic alterations affecting WSS in BAV patients can be assessed based on 4D-flow acquisitions, but reference values for healthy and disease states are yet undefined (Potters et al., 2014) and cannot be used for risk stratification.

To address these limitations, we developed and tested two new methods to improve the reliability of WSS computation from 4Dflow discrete velocity field. These are based on a fully volumetric and a local planar analysis, respectively. To test these methods, computational fluid-dynamics (CFD) models of the thoracic aorta with healthy and bicuspid aortic valve were created, accounting for the complex anatomy of the domain. To mimic MR acquisitions, the computed velocity fields were down-sampled to obtain arti-ficial $4 \mathrm{D}$-flow datasets. The sensitivity of our methods to data spatial resolution and noise was assessed. Finally, we applied our methods on 4D-flow datasets of three healthy subjects and three BAV patients, assessing consistent hemodynamic differences and shear stress patterns.

a

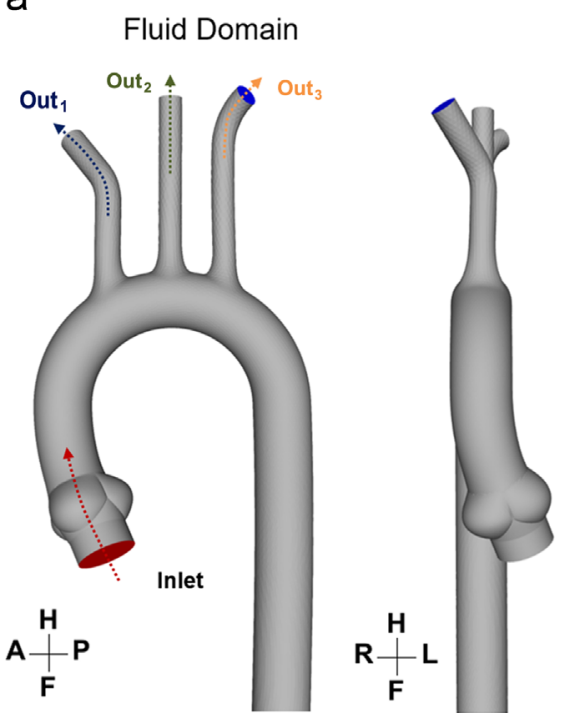

\section{Material and methods}

\subsection{Artificial $4 D$-flow datasets}

Two geometrical models of the thoracic aorta with open physiological tricuspid (TAV) and bicuspid aortic valve (BAV), respectively, were borrowed from previous work (Conti et al., 2010a, 2010b; Della Corte et al., 2012) (Fig. 1.a,b). Transient CFD simulations were run with the commercial solver Fluent (Ansys Inc., Canonsburg, PA, USA). Time-dependent flow rates were imposed at the aorta inlet and at the supra-aortic branches (Fig. 1c), zero-pressure was set at the aorta outlet, and no-slip condition at the wall was assumed. Blood was modeled as an incompressible Newtonian fluid $\left(\mu=3.7 \mathrm{cP}, \rho=1060 \mathrm{~kg} / \mathrm{m}^{3}\right)$. Artificial 4D-flow datasets were obtained for the two models with reference to peak systole following our previously published approach (Morbiducci et al., 2012). Briefly, CFD-derived velocity fields were downsampled with an isotropic spatial resolution, obtaining a voxel grid. In order to mimic MR spatial regularization, the velocity data associated to each voxel were computed as the weighted average of the CFD velocity data at the points falling within the voxel, where the weights were generated through a Gaussian function of the distance between these points and the voxel center. This procedure was implemented with a spatial resolution $(\Delta x)$ of $1,2,3$ and $4 \mathrm{~mm}$.

On the TAV and BAV datasets with a $2 \mathrm{~mm}$ spatial resolution, which is consistent with current clinical practice (Dyverfeldt et al., 2015), we emulated the definition of sub optimal encoding velocity (VENC) values to account for MR noise. Gaussian noise with a variance related to the VENC was added to the dataset, thus worsening the signal-to-noise ratio (Petersson et al., 2012). For each dataset, three noisy variants were obtained, by adding noise consistent with VENC values exceeding the actual maximum velocity by $5 \%, 10 \%$ and $20 \%$, respectively.

\subsection{Real $4 D$-flow datasets}

Three BAV-affected patients without complex congenital heart diseases (2 males, age 19,28,31) were recruited prospectively. Three healthy volunteers (3 males, age $17,23,25$ ) were enrolled as controls. 4D-flow acquisitions were performed at John Radcliffe Hospital (Oxford, United Kingdom). Flow-sensitive gradient-echo pulse sequences were acquired with prospective ECG-gating during freebreathing, using a respiratory navigator, on a 3.0 T Magnetom Trio MR system (Siemens, Erlangen, Germany). The acquisition volume was oriented along an oblique-sagittal plane encompassing the whole thoracic aorta, as well as the ascending aorta and the aortic arch. 4D-flow sequences were set with the following specifics: i) voxel sizing $=1.67 \div 2.2 \mathrm{~mm}^{3}$; ii) $\mathrm{VENC}=150 \div 370 \mathrm{~cm} / \mathrm{s}$; iii) Flip Angle $=7^{\circ}$; iv) Echo Time $=2.3 \div 2.5 \mathrm{~ms} ; \mathrm{v}$ ) Repetition Time $=40 \mathrm{~ms}$. The Institu-tional Review Board approved the study and informed consent was obtained from each participant. The time-frame with the highest velocity-to-noise ratio was chosen as the most representative of peak systole and selected for post-processing.

\subsection{Definition of the region of interest (ROI)}

The region of interest (ROI) in the datasets consisted in the aortic lumen. On CFDderived artificial 4D-flow datasets, voxels within the ROI were automatically

\section{b}

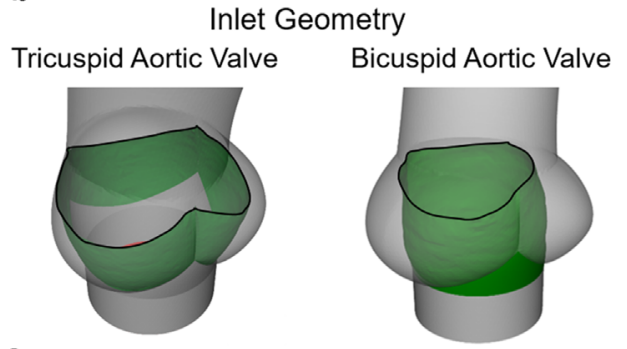

C

Flow rate boundary conditions

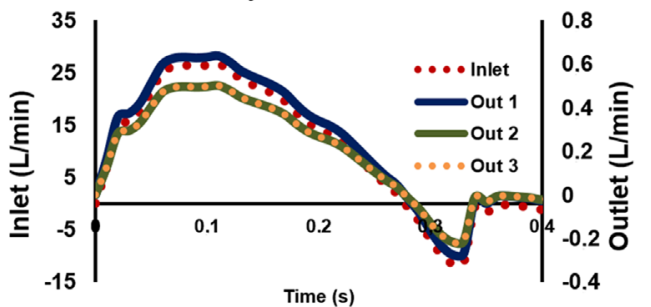

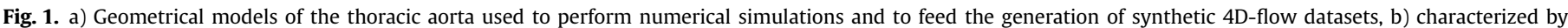
physiological tricuspid (TAV) and bicuspid aortic valve (BAV). c) Flow rate waveforms applied as boundary conditions. 
a
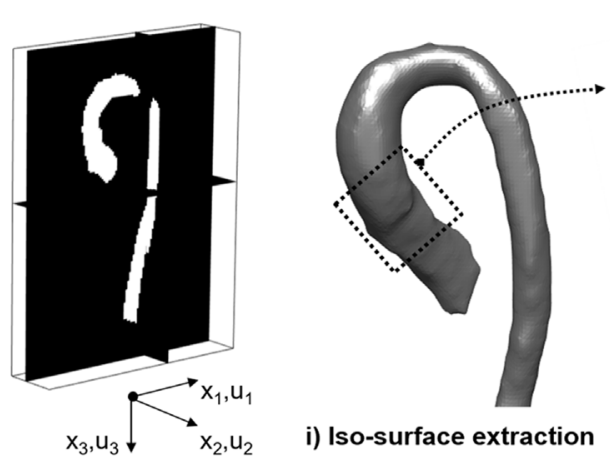

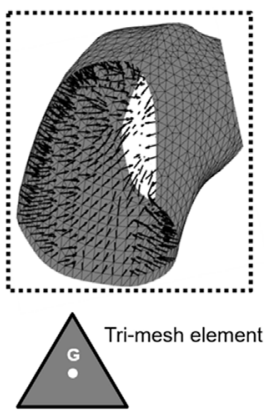

ii) Face normals

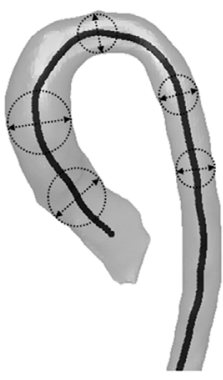

iii) Centerline definition

b

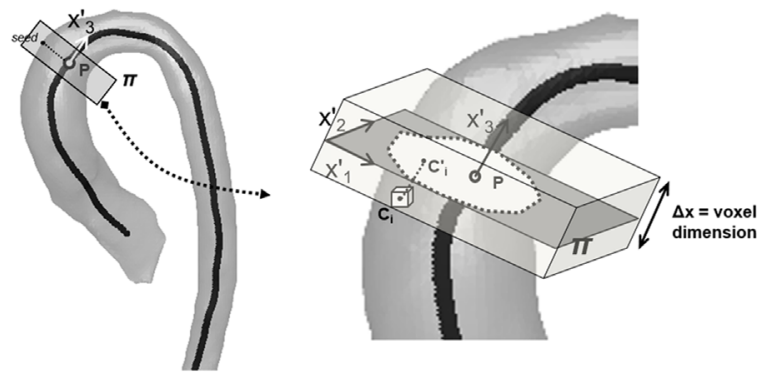

iv) Cross-section computation

C

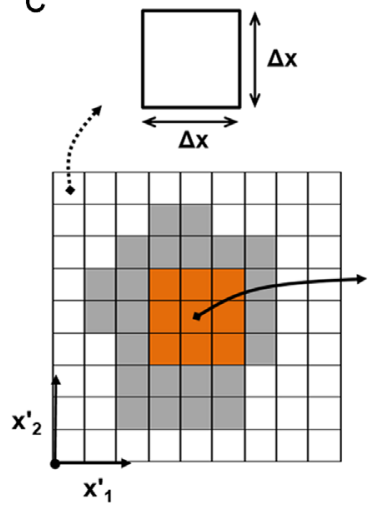

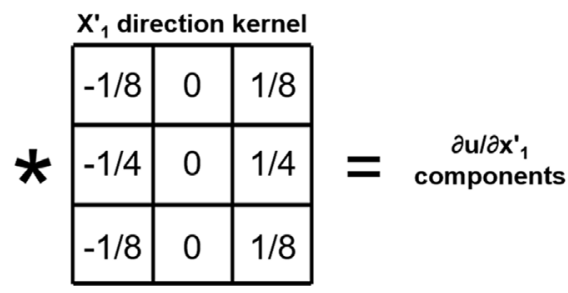

\begin{tabular}{|c|c|c|}
\hline$(-\Delta x, 0)$ & $(0,0)$ & $(\Delta \mathrm{x}, 0)$ \\
\hline$\underset{(-\Delta x,-\Delta x)}{u}$ & $\begin{array}{c}u \\
(0,-\Delta x)\end{array}$ & $\underset{(\Delta \mathbf{x},-\Delta \mathbf{x})}{\mathbf{u}}$ \\
\hline
\end{tabular}

\begin{tabular}{|c|c|c|}
\hline \multicolumn{3}{|c|}{$\mathrm{X}_{2}{ }_{2}$ direction kerne } \\
\hline $1 / 8$ & $1 / 4$ & $1 / 8$ \\
\hline 0 & 0 & 0 \\
\hline$-1 / 8$ & $-1 / 4$ & $-1 / 8$ \\
\hline
\end{tabular}

$=\begin{gathered}\partial u / \partial x_{2}^{\prime} \\ \text { components }\end{gathered}$

$\partial \mathrm{u}_{1} / \partial \mathrm{x}_{1}{ }_{1}(0,0)=1 / 4^{*}\left[\mathrm{u}_{1}(\Delta \mathrm{x}, 0)-\mathrm{u}_{1}(-\Delta \mathrm{x}, 0)\right]+1 / 8^{*}\left[\mathrm{u}_{1}(\Delta \mathrm{x}, \Delta \mathrm{x})\right.$

$\left.+\mathrm{u}_{1}(\Delta \mathrm{x},-\Delta \mathrm{x})-\mathrm{u}_{1}(-\Delta \mathrm{x}, \Delta \mathrm{x})-\mathrm{u}_{1}(-\Delta \mathrm{x},-\Delta \mathrm{x})\right] / \Delta \mathrm{x}$

$=\quad \partial \mathrm{u}_{2} / \partial \mathrm{x}_{1}^{\prime}(0,0)=1 / 4^{*}\left[\mathrm{u}_{2}(\Delta \mathrm{x}, 0)-\mathrm{u}_{2}(-\Delta \mathrm{x}, 0)\right]+1 / 8^{*}\left[\mathrm{u}_{2}(\Delta \mathrm{x}, \Delta \mathrm{x})\right.$

$\left.+\mathrm{u}_{2}(\Delta \mathrm{x},-\Delta \mathrm{x})-\mathrm{u}_{2}(-\Delta \mathrm{x}, \Delta \mathrm{x})-\mathrm{u}_{2}(-\Delta \mathrm{x},-\Delta \mathrm{x})\right] / \Delta \mathrm{x}$

$\partial u_{3} / \partial x_{1}^{\prime}(0,0)=1 / 4^{*}\left[u_{3}(\Delta x, 0)-u_{3}(-\Delta x, 0)\right]+1 / 8^{*}\left[u_{3}(\Delta x, \Delta x)\right.$

$\left.+\mathrm{u}_{3}(\Delta \mathrm{x},-\Delta \mathrm{x})-\mathrm{u}_{3}(-\Delta \mathrm{x}, \Delta \mathrm{x})-\mathrm{u}_{3}(-\Delta \mathrm{x},-\Delta \mathrm{x})\right] / \Delta \mathrm{x}$

$\partial u / \partial x_{2}^{\prime}$

$\partial \mathrm{u}_{1} / \partial \mathrm{x}_{2}^{\prime}(0,0)=1 / 4^{*}\left[\mathrm{u}_{1}(0, \Delta \mathrm{x})-\mathrm{u}_{1}(0,-\Delta \mathrm{x})\right]+1 / 8^{*}\left[\mathrm{u}_{1}(-\Delta \mathrm{x}, \Delta \mathrm{x})\right.$

$\left.+\mathrm{u}_{1}(\Delta \mathrm{x}, \Delta \mathrm{x})-\mathrm{u}_{1}(-\Delta \mathrm{x},-\Delta \mathrm{x})-\mathrm{u}_{1}(\Delta \mathrm{x},-\Delta \mathrm{x})\right] / \Delta \mathrm{x}$

$=\quad \partial \mathrm{u}_{2} / \partial \mathrm{x}_{2}^{\prime}(0,0)=1 / 4^{\star}\left[\mathrm{u}_{2}(0, \Delta \mathrm{x})-\mathrm{u}_{2}(0,-\Delta \mathrm{x})\right]+1 / 8^{\star}\left[\mathrm{u}_{2}(-\Delta \mathrm{x}, \Delta \mathrm{x})\right.$

$\left.+\mathrm{u}_{2}(\Delta \mathrm{x}, \Delta \mathrm{x})-\mathrm{u}_{2}(-\Delta \mathrm{x},-\Delta \mathrm{x})-\mathrm{u}_{2}(\Delta \mathrm{x},-\Delta \mathrm{x})\right] / \Delta \mathrm{x}$

$\partial u_{3} / \partial x_{2}^{\prime}(0,0)=1 / 4^{*}\left[u_{3}(0, \Delta x)-u_{3}(0,-\Delta x)\right]+1 / 8^{*}\left[u_{3}(-\Delta x, \Delta x)\right.$ $\left.+\mathrm{u}_{3}(\Delta \mathrm{x}, \Delta \mathrm{x})-\mathrm{u}_{3}(-\Delta \mathrm{x},-\Delta \mathrm{x})-\mathrm{u}_{3}(\Delta \mathrm{x},-\Delta \mathrm{x})\right] / \Delta \mathrm{x}$

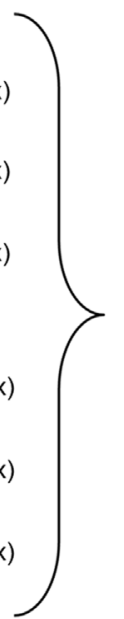

Strain rate tensor

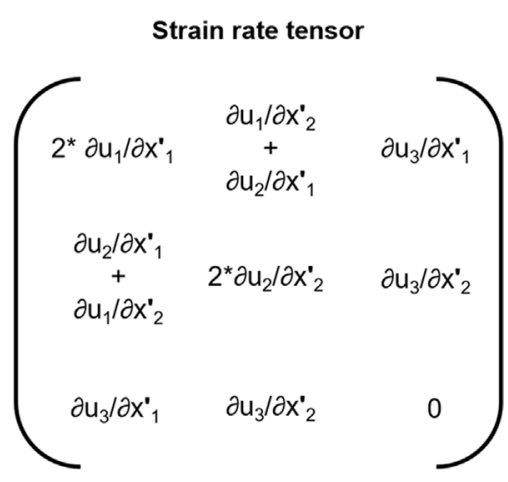

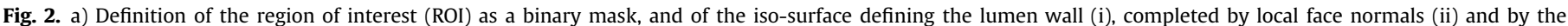

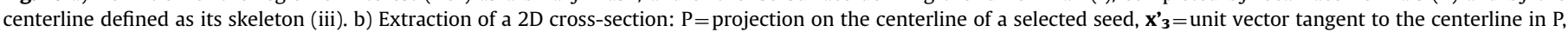

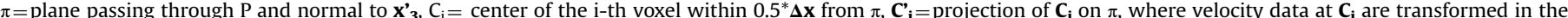

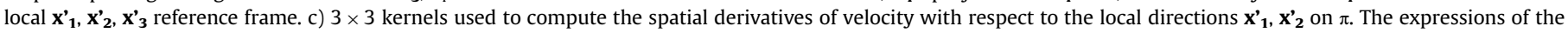
strain tensor components as obtained through the use of the kernels are reported. 
defined as those containing at least one point with associated CFD velocity. On real datasets, the velocity magnitude volume was derived combining the information of the three encoded scalar velocity components, and on the para-sagittal slices the aortic lumen was manually traced to define the ROI.

In both cases, a 3D binary mask was obtained: values equal to 1 were associated to voxels inside the ROI and 0 otherwise. Subsequently, the wall and the centerline of the aorta were automatically defined (Fig. 2a). The wall was identified as the iso-surface encompassing the ROI, which was smoothed through laplacian diffusion (Desbrun et al., 1999) to obtain a regularized surface mesh of triangular elements; inward unitary face normals were computed. The centerline was obtained through skeletonization of the ROI (Shih, 2010); the points defining the skeleton were ordered end-to-end via Dijkstra algorithm (Dijkstra, 1959).

\subsection{Wall shear stress computation from $4 D$-flow data}

Under the assumption of Newtonian blood behavior, the shear components of the stress tensor can be obtained through the spatial derivatives of the velocity field $\mathbf{u}(\mathbf{x})$ as:

$\tau_{i j}=\mu\left(\frac{\partial u_{i}}{\partial x_{j}}+\frac{\partial u_{j}}{\partial x_{i}}\right)$ with $i \neq j$

The computation of $\tau_{i j}$ at the ROI boundary in a reference frame that is consistent with the local geometry yields the wall shear stress (WSS) (Petersson et al., 2012).

On 4D-flow discrete $\mathbf{u}(\mathbf{x})$ data, derivatives were computed numerically. Owing to the almost isotropic spatial resolution of the data, a weighted central difference scheme was adopted through Sobel filters (Shih, 2010). In this work, we used two different approaches to compute the stress tensor and the WSS: Local Planar (LP) and Global Volumetric (GV), each one exploiting a different variant of the Sobel filter.

The LP approach operated on 2D planes and consisted of five steps:

i) definition within the 3D ROI of a 2D cross-section, starting from a user-defined seed placed on the surface of the ROI. The seed's projection on the aortic centerline (P) and the corresponding tangent unit vector $\mathbf{x}_{\mathbf{3}}$ were automatically computed, and defined the cross-sectional plane $\pi$ passing through $\mathbf{P}$ and normal to $\mathbf{x}_{\mathbf{3}}$ (Fig. 2b).

ii) Selection of the voxels whose center was within $0.5^{*} \Delta x$ from $\pi$. For each of these voxels, the corresponding three velocity components were assigned to the projection of the voxel center on $\pi$ and transformed in the local ortho-normal coordinate system $\left(\mathrm{x}_{1}^{\prime}, \mathrm{x}_{2}, \mathrm{x}_{3}{ }_{3}\right)$ aligned with $\pi$ (Fig. $2 \mathrm{~b}$ ).

iii) Convolution of two $3 \times 3$ kernels to compute derivatives in the $x_{1}{ }_{1}$ and $x_{2}$ directions, respectively, to obtain the spatial derivatives $\partial \boldsymbol{u} / \partial x_{1}^{\prime}$ and $\partial \boldsymbol{u} / \partial x_{2}^{\prime}$ at each point on $\pi$ (Fig. $2 \mathrm{c}$ ).

iv) Removal of boundary artifacts. Since $3 \times 3$ kernels were used, only the deriva-tives' values located at least three pixels far from the aortic wall were maintained and interpolated through cubic splines along the $\mathrm{x}_{1}{ }_{1}$ and $\mathrm{x}_{2}$ directions. The values at the remaining near-wall pixels were recomputed by spline sampling.

v) Computation of $\tau_{i j}$, under the assumption $\partial u_{i} / \partial x_{3}^{\prime}=0 \forall i$

The GV approach directly operated on the volumetric dataset by means of three $3 \times 3 \times 3$ kernels (Fig. 3 ), which expanded the concept of $2 \mathrm{D}$ kernels. This approach consisted of four steps:

i) convolution of the three kernels, for the global $x_{1}, x_{2}, x_{3}$ directions, respectively, on the raw velocity data, obtaining the spatial derivatives $\partial \boldsymbol{u} / \partial \boldsymbol{x}$ at each point of the 3D ROI.

ii) Sampling of the derivatives' values on the aortic wall. For each triangular element of the wall triangulated surface, the center of mass $\mathbf{G}$ was considered. The values of $\partial \boldsymbol{u} / \partial \boldsymbol{x}$ falling within two voxels from $\mathbf{G}$ were interpolated through cubic splines along the $\mathrm{x}_{1}, \mathrm{x}_{2}, \mathrm{x}_{3}$ directions. Splines were sampled at $\mathbf{G}$ to evaluate the corresponding value of $\partial \boldsymbol{u} /\left.\partial \boldsymbol{x}\right|_{\boldsymbol{x}=\boldsymbol{x}_{G}}$.

iii) Rigid rotation of $\partial \boldsymbol{u} / \partial \boldsymbol{x}$ into a local orthonormal reference frame $\mathrm{t}_{1}, \mathrm{t}_{2}, \mathrm{n}$, where $\mathrm{n}$ is the unit vector obtained averaging the normal to the wall at the nodes defining the triangular element, while $t_{1}$ and $t_{2}$ are normal to $n$ and oriented along the axial and circumferential direction of the vessel, respectively.

iv) Computation of the WSS magnitude based on the derivatives of components $\mathrm{u}_{\mathrm{t} 1}$ and $\mathrm{u}_{\mathrm{t} 2}$ with respect to $\mathrm{n}$ and visualization of the full 3D WSS contour.

\subsection{Artificial datasets analysis}

CFD-derived artificial datasets were processed through the LP and GV methods and the corresponding WSS values were compared to the ones computed directly from CFD simulations. Two sets of tests were performed. In the first set, we tested the dependency on the dataset's spatial resolution of the LP and GV methods, by pro-cessing CFD data sampled at different spatial resolutions (Table 1). For the GV method, the capability to correctly capture the 3D space-distribution of WSS was analyzed. For both methods, the capability to correctly quantify peak WSS values on a

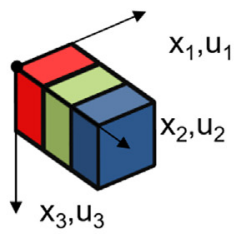

\section{$\mathrm{X}_{1}$ direction kernel}

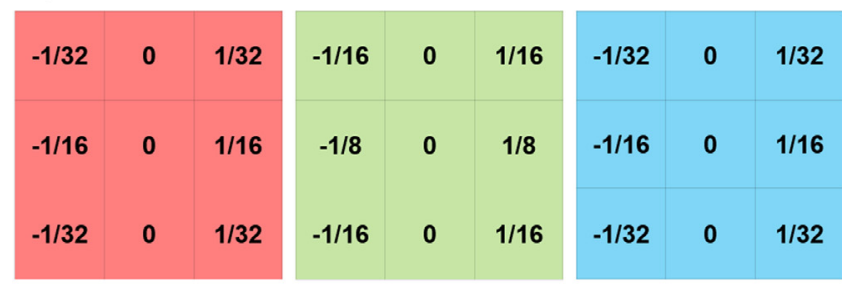

\section{$X_{2}$ direction kernel}

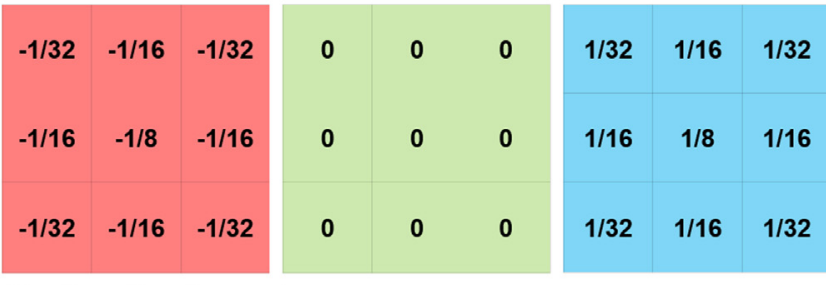

\section{$\mathrm{X}_{3}$ direction kernel}

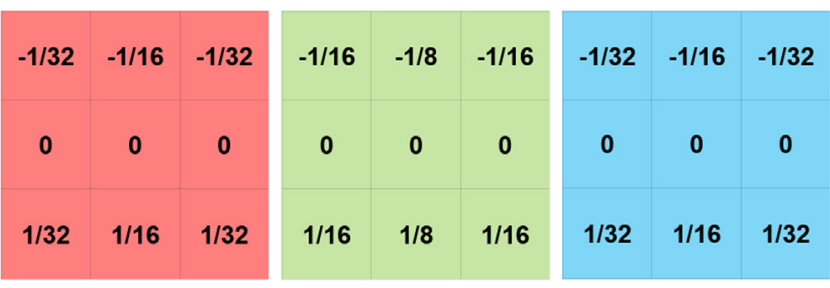

Fig. 3. $3 \times 3 \times 3$ kernels used when computing the spatial derivatives of velocities in a fully 3D approach. Top panel: the kernel is represented as it is actually oriented in the 3D space, and a color code is used to identify the three layers of the kernel each one being a $3 \times 3$ matrix. Bottom panel: the weights within each layer of the 3D kernel are reported. These change depending on whether the kernel is applied along the $x_{1}, x_{2}$, or $x_{3}$ direction, so that the orientation of the kernel is not changed. (For interpretation of the references to color in this figure legend, the reader is referred to the web version of this article.)

Table 1

Specifics of the numerical experiments.

\begin{tabular}{|c|c|c|c|c|}
\hline \multicolumn{5}{|l|}{ CFD domains } \\
\hline & 1 - TAV & \multicolumn{2}{|l|}{$2-\mathrm{BAV}$} \\
\hline & Peak flow rate $(\mathrm{L} / \mathrm{min})$ & 26.7 & \multicolumn{2}{|l|}{26.7} \\
\hline \multicolumn{2}{|c|}{ Peak velocity $(\mathrm{cm} / \mathrm{s})$} & 117.4 & \multicolumn{2}{|l|}{144.2} \\
\hline \multicolumn{2}{|c|}{$\begin{array}{l}\text { Cells characteristic dimension } \\
(\mathrm{mm})\end{array}$} & 0.48 & \multicolumn{2}{|l|}{0.48} \\
\hline \multicolumn{2}{|c|}{ Approximate number of cells } & $1.1 \cdot 10^{6}$ & \multicolumn{2}{|l|}{$1.1 \cdot 10^{6}$} \\
\hline \multicolumn{5}{|l|}{ Numerical experiments } \\
\hline \multicolumn{5}{|l|}{ First set of experiments } \\
\hline $\begin{array}{l}\text { Voxel linear dimension } \\
\quad(\mathrm{mm})\end{array}$ & 1 & 2 & 3 & 4 \\
\hline $\begin{array}{l}\text { Approximate number of } \\
\text { voxels }\end{array}$ & $270 \cdot 10^{3}$ & $40 \cdot 10^{3}$ & $13 \cdot 10^{3}$ & $6 \cdot 10^{3}$ \\
\hline $\operatorname{VENC}(\mathrm{cm} / \mathrm{s})$ & 112.0 & & 126.6 & \\
\hline \multicolumn{5}{|l|}{$\begin{array}{l}\text { Second set of } \\
\text { experiments }\end{array}$} \\
\hline Voxel dimension (mm) & 2 & & 2 & \\
\hline $\operatorname{VENC}(\mathrm{cm} / \mathrm{s})(+$ noise $)$ & $112.0(+$ & $-5 \%,+10 \%,+20 \%)$ & $\begin{array}{l}126.6(+ \\
+20 \%)\end{array}$ & $+10 \%$, \\
\hline
\end{tabular}

section of interest was assessed; in particular, for each simulated condition, i.e. TAV and BAV, we referred to a cross-section of the ascending aorta at high WSS regions as identified by CFD simulations. Moreover, the effect of spatial resolution on peak WSS 
a

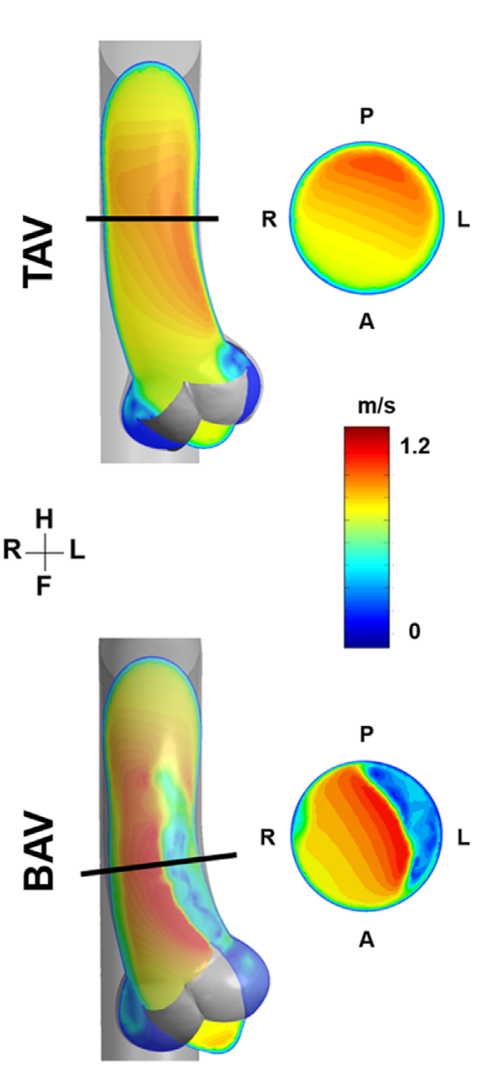

$b$

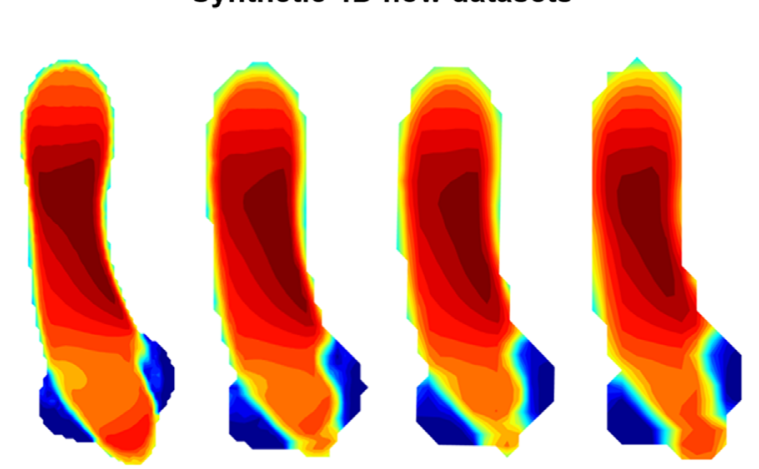

$$
\Delta \mathrm{x}=1 \mathrm{~mm} \quad \Delta \mathrm{x}=2 \mathrm{~mm} \quad \Delta \mathrm{x}=3 \mathrm{~mm} \quad \Delta \mathrm{x}=4 \mathrm{~mm}
$$

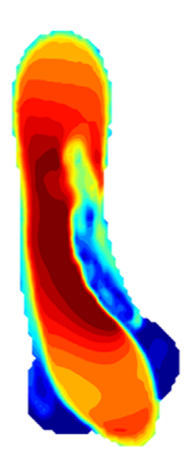

C

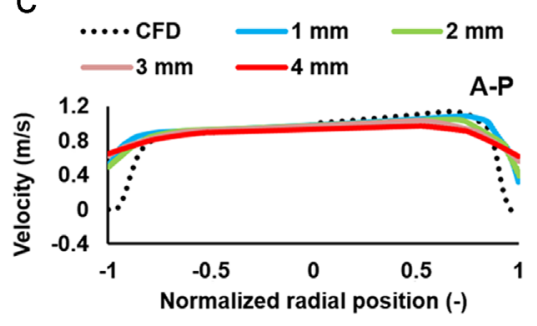

L-R
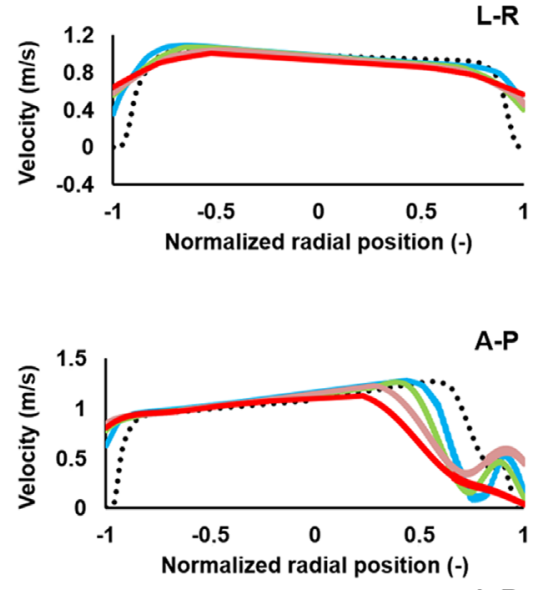

L-R

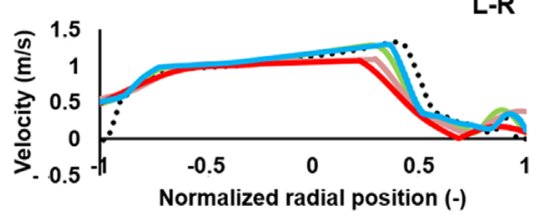

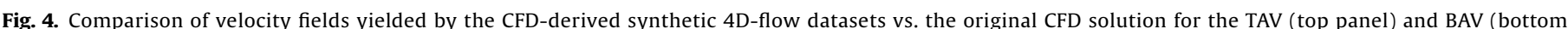

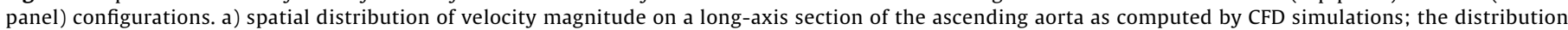

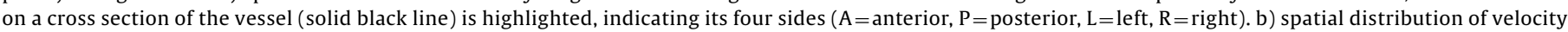

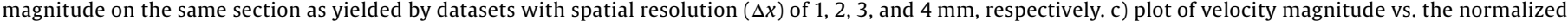

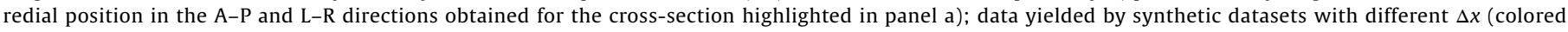

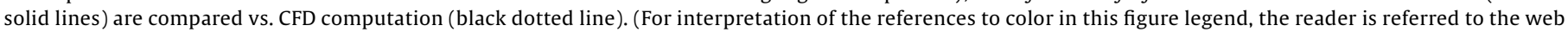
version of this article.)

quantification was assessed when integrating the GV and LP methods as it should be done in a hypothetical clinical setting, where no CFD solution would be available to guide the selection of the section to be analyzed. In this case, a two-step approach was followed: i) extraction of the 3D map of WSS from the raw data through the GV method and identification of the peak WSS region; ii) definition of a cross-section at this region and quantification of peak WSS on the section's boundary.

In the second set of tests, we tested the sensitivity of our methods to noise. The above described analysis was performed on each noisy variant of the datasets with a $2 \mathrm{~mm}$ spatial resolution.

\subsection{Real datasets analysis}

4D-flow datasets were analyzed by integrating the LP and GV methods as previously described.

\section{Results}

\subsection{Processing of synthetic datasets: sensitivity to spatial resolution}

\subsubsection{Velocity field}

For every spatial resolution considered, the overall spatial distribution of velocity magnitude within the ROI closely resembled the one computed directly through CFD TAV and BAV models (Fig. 4a and b). No macroscopic effect on bulk flow was observed: in particular, peak velocities changed by less than $15 \%$ and acceptable imbalances in blood mass $(<5 \%)$ between the ROI inflow and outflows were computed. Instead, near-wall velocity values and patterns were markedly affected by spatial resolution, and near-wall secondary flows in BAV could be captured only when a spatial resolution of 1 or $2 \mathrm{~mm}$ was used (Fig. 4c).

\subsubsection{WSS 3D pattern}

The WSS 3D distribution yielded by the GV method was qualitatively consistent with the one computed directly through CFD simulations, in terms of location of hotspots and extent of high WSS regions, when artificial datasets with resolutions of 1 and 2 $\mathrm{mm}$ were considered (Fig. 5b).

\subsubsection{Peak WSS quantification}

The cross-sections selected on the TAV and BAV configurations are indicated in Fig. 5a. CFD simulations computed a peak and mean WSS on the boundary of the cross section equal to 10.26 and 6.14 Pa, respectively, for the TAV model, and equal to 14.43 and 9.04 Pa, respectively, in the BAV model.

On artificial datasets, on these same cross-sections, the GV and LP methods captured the WSS increase in BAV datasets as compared to TAV ones independently of the dataset's space resolution, but both methods largely underestimated WSS values in both scenarios (Table 2). For the GV method, such underestimation decreased almost linearly with the improvement in dataset's spatial resolution ( $r=0.92$ and 0.87 for TAV and BAV datasets, respectively). Still, peak WSS was $0.98 \mathrm{~Pa}$ ( $-90.5 \%$ vs. CFD) and 1.07 $\mathrm{Pa}(-92.6 \%)$ when processing TAV and BAV datasets with a 
a
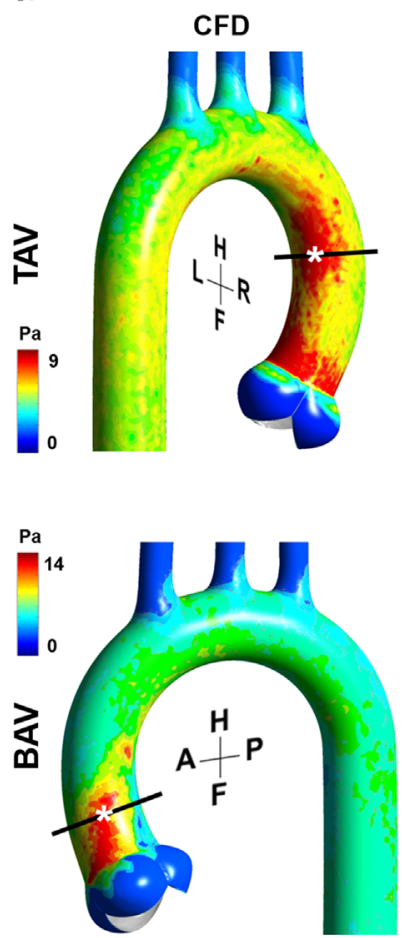

b

\author{
i)
}
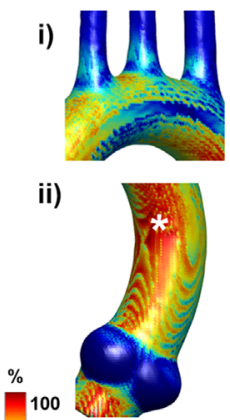

$\Delta \mathrm{x}=1 \mathrm{~mm}$

i)

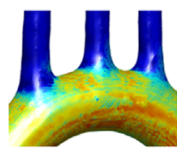

ii)

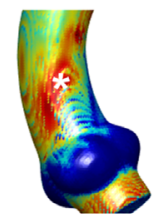

Synthetic 4D flow datasets
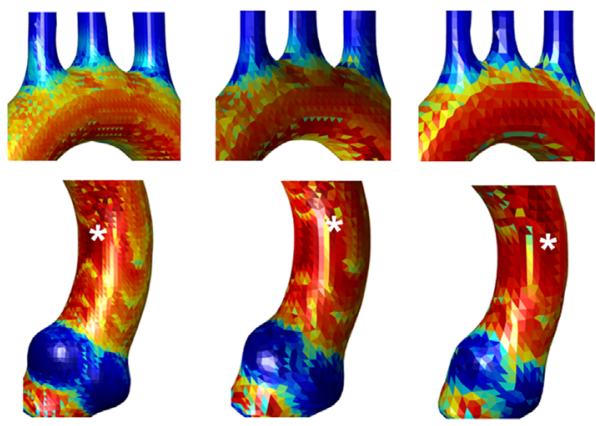

$\Delta \mathrm{x}=2 \mathrm{~mm}$

$\Delta \mathrm{x}=3 \mathrm{~mm}$

$\Delta \mathrm{x}=4 \mathrm{~mm}$
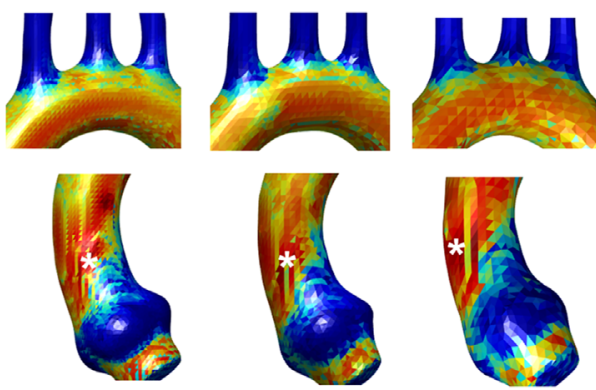

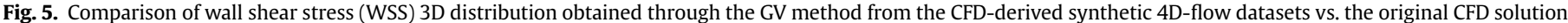

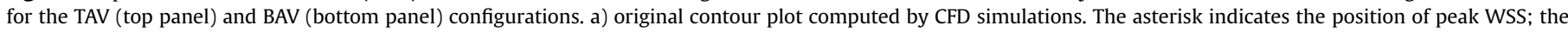

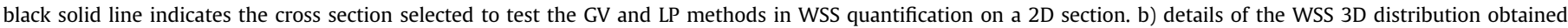
through the GV method on the aortic arch (i) and on the ascending aorta (ii) from datasets with spatial resolution ( $\Delta x$ ) of $1,2,3$, and 4 mm, respectively.

Table 2

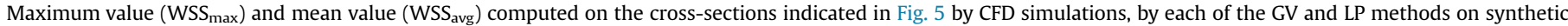
datasets with different spatial resolutions $(\Delta x)$, and by the integration of GV and LP methods.

\begin{tabular}{|c|c|c|c|c|c|}
\hline & & \multicolumn{2}{|l|}{ TAV } & \multicolumn{2}{|l|}{ BAV } \\
\hline & & $\mathrm{WSS}_{\max }[\mathrm{Pa}]$ & $\mathrm{WSS}_{\text {avg }}[\mathrm{Pa}]$ & $\mathrm{WSS}_{\max }[\mathrm{Pa}]$ & WSS $_{\text {avg }}[\mathrm{Pa}]$ \\
\hline CFD & & 10.26 & 6.52 & 14.43 & 9.04 \\
\hline \multirow[t]{4}{*}{ GV method } & $\Delta x=1 \mathbf{m m}$ & 1.71 & 1.27 & 2.19 & 1.41 \\
\hline & $\Delta \mathbf{x}=\mathbf{2} \mathbf{m m}$ & 0.98 & 0.75 & 1.07 & 0.70 \\
\hline & $\Delta x=3 m m$ & 0.63 & 0.51 & 0.68 & 0.48 \\
\hline & $\Delta x=4 m m$ & 0.45 & 0.39 & 0.49 & 0.32 \\
\hline \multirow[t]{5}{*}{ LP method } & $\Delta x=1 \mathbf{m m}$ & 4.50 & 1.74 & 5.97 & 2.15 \\
\hline & $\Delta \mathbf{x}=\mathbf{2 m m}$ & 1.79 & 0.93 & 2.77 & 1.11 \\
\hline & $\Delta \mathbf{x}=3 \mathrm{~mm}$ & 1.10 & 0.64 & 1.35 & 0.58 \\
\hline & $\Delta x=4 \mathrm{~mm}$ & 0.64 & 0.46 & 0.81 & 0.38 \\
\hline & $\Delta x=1 \mathbf{m m}$ & 4.16 & 1.67 & 5.66 & 2.25 \\
\hline \multirow[t]{3}{*}{ GV $+\mathbf{L P}$ integration } & $\Delta \mathbf{x}=\mathbf{2} \mathbf{m m}$ & 1.66 & 1.05 & 2.74 & 1.13 \\
\hline & $\Delta \mathbf{x}=3 \mathrm{~mm}$ & 1.03 & 0.66 & 1.17 & 0.59 \\
\hline & $\Delta x=4 m m$ & 0.91 & 0.51 & 0.95 & 0.37 \\
\hline
\end{tabular}

$2 \mathrm{~mm}$ spatial resolution. These values increased to only $1.71 \mathrm{~Pa}$ ( $-83.3 \%$ vs. CFD) and $2.19 \mathrm{~Pa}(-84.8 \%)$, respectively, when $1 \mathrm{~mm}$ spatial resolution was adopted. The LP method provided higher WSS values, and the underestimation of WSS decreased quadratically with the improvement in spatial resolution $(r=0.98$ and 0.99 for TAV and BAV datasets, respectively). When processing datasets with a $2 \mathrm{~mm}$ spatial resolution, peak WSS was $1.79 \mathrm{~Pa}$ $(-82.5 \%$ vs. CFD) and $2.77 \mathrm{~Pa}(-80.8 \%)$ for the TAV and BAV configurations. When a $1 \mathrm{~mm}$ spatial resolution was adopted, these values were $4.5 \mathrm{~Pa}(-56.1 \%)$ and 5.97 $\mathrm{Pa}(-58.6 \%)$.

3.1.4. Peak WSS quantification by integrating the GV and LP methods

The section of interest identified based on the WSS map yielded by the GV method was consistent with the section selected based on CFD results. On progressively coarser TAV datasets, the former was tilted by $0.6,13.0,10.5$, and $14.2^{\circ}$ with respect to the latter, and the distance between the respective intersections with the vessel centerline was 2.0, 8.0, 6.7 and $9.8 \mathrm{~mm}$. On progressively coarser BAV datasets, the difference in section orientation was $0.2,1.1,3.3$ and $3.9^{\circ}$, and the maximum distance between the respective intersections with the aortic wall was 1.0, 6.6, 7.3 and $4 \mathrm{~mm}$. Accordingly, WSS peak and mean values quantified by integrating the GV and LP methods closely matched the ones computed by the LP method alone after exploiting CFD results to select the section of interest (Table 2). Of note, the position of the peak WSS was always consistent with the position of the WSS hotspots highlighted by the 3D WSS map obtained through the GV method (Fig. 6). 

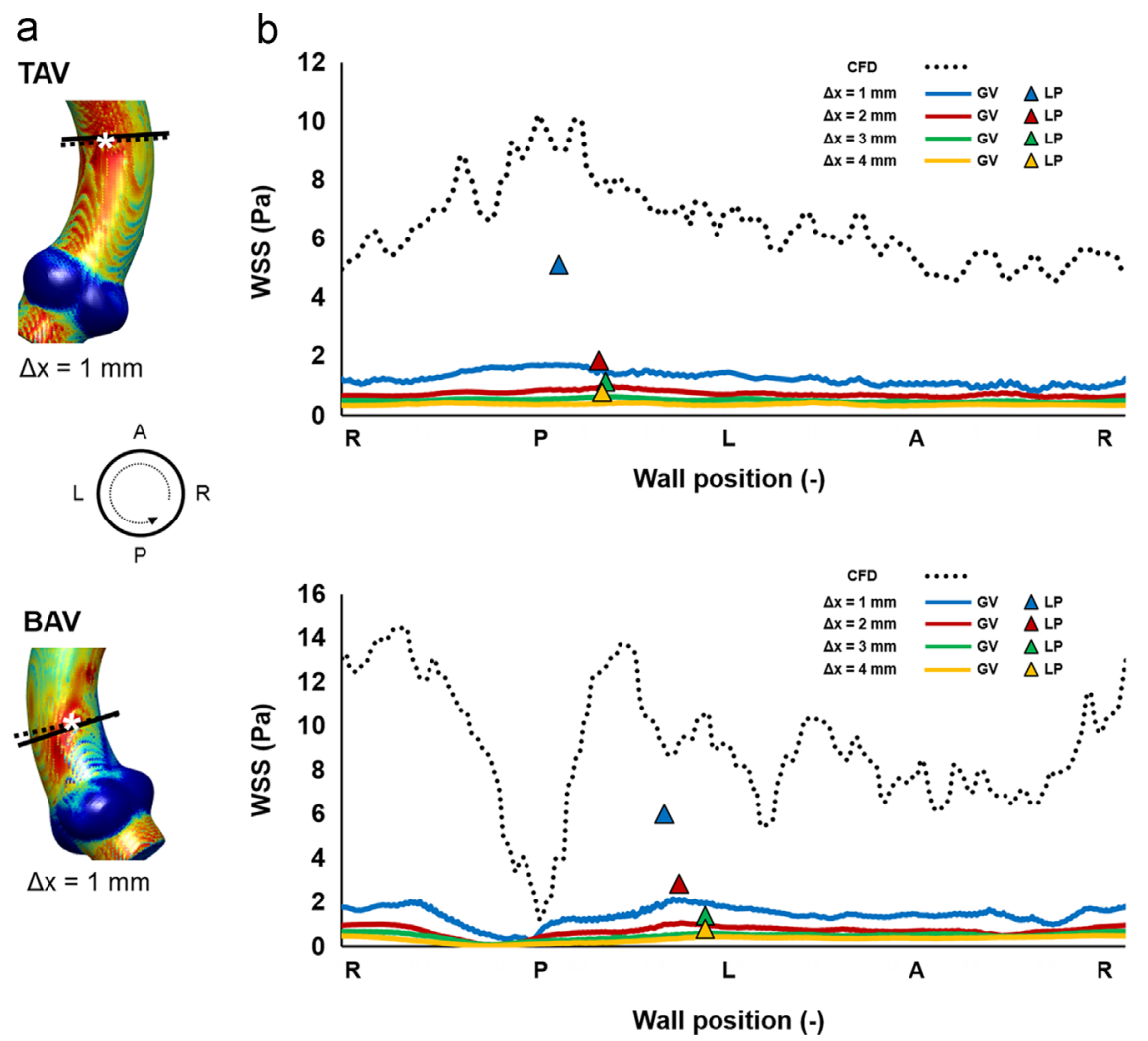

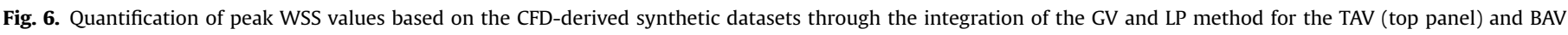

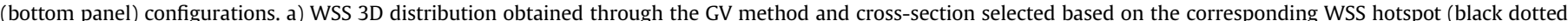

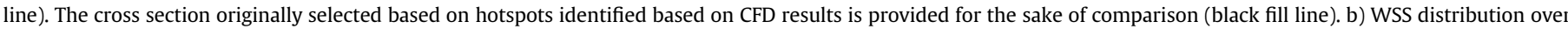

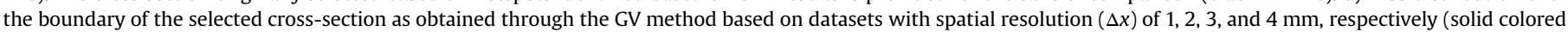

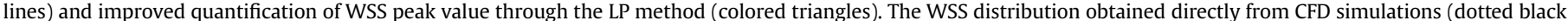
line) is reported for the sake of comparison. (For interpretation of the references to color in this figure legend, the reader is referred to the web version of this article.)

\section{Table 3}

Maximum value $\left(\right.$ WSS $_{\max }$ ) and mean value $\left(\mathrm{WSS}_{\mathrm{avg}}\right)$ computed on the cross-sections indicated in Fig. 5 by CFD simulations, by each of the GV and LP methods on synthetic datasets with spatial resolution equal to $\Delta x=2 \mathrm{~mm}$ and variable noise levels of $5 \%, 10 \%, 20 \%$ related to sub-optimal VENC definition. Percentage variations with respect to results obtained without noise are reported in brackets.

\begin{tabular}{|c|c|c|c|c|c|}
\hline & & \multicolumn{2}{|l|}{ TAV } & \multicolumn{2}{|l|}{ BAV } \\
\hline & & $\mathrm{WSS}_{\max }[\mathrm{Pa}]$ & $\begin{array}{l}\text { WSS }_{\text {avg }} \\
\text { [Pa] }\end{array}$ & $\mathrm{WSS}_{\max }[\mathrm{Pa}]$ & $\begin{array}{l}\text { WSS }_{\text {avg }} \\
\text { [Pa] }\end{array}$ \\
\hline \multirow[t]{3}{*}{ GV method } & $\begin{array}{l}\mathbf{5 \%} \\
\text { noise }\end{array}$ & $0.95(-3.1 \%)$ & $\begin{array}{l}0.76 \\
(+1.3 \%)\end{array}$ & $1.12(+4.7 \%)$ & $\begin{array}{l}0.74 \\
(+5.7 \%)\end{array}$ \\
\hline & $\begin{array}{l}10 \% \\
\text { noise }\end{array}$ & $0.95(-3.1 \%)$ & $\begin{array}{l}0.76 \\
(+1.3 \%)\end{array}$ & $1.11(+3.7 \%)$ & $\begin{array}{l}0.75 \\
(+7.1 \%)\end{array}$ \\
\hline & $\begin{array}{l}20 \% \\
\text { noise }\end{array}$ & $0.94(-4.1 \%)$ & $\begin{array}{l}0.77 \\
(+2.7 \%)\end{array}$ & $1.08(+0.9 \%)$ & $\begin{array}{l}0.74 \\
(+5.7 \%)\end{array}$ \\
\hline \multirow[t]{3}{*}{ LP method } & $\begin{array}{l}5 \% \\
\text { noise }\end{array}$ & $1.79(+0.0 \%)$ & $\begin{array}{l}0.95 \\
(+2.1 \%)\end{array}$ & $2.69(-2.9 \%)$ & $\begin{array}{l}1.18 \\
(+6.3 \%)\end{array}$ \\
\hline & $\begin{array}{l}10 \% \\
\text { noise }\end{array}$ & 1.87 (4.5\%) & $\begin{array}{l}1.01 \\
(+8.6 \%)\end{array}$ & $3.00(+8.3 \%)$ & $\begin{array}{l}1.19 \\
(+7.2 \%)\end{array}$ \\
\hline & $\begin{array}{l}20 \% \\
\text { noise }\end{array}$ & $1.83(+2.2 \%)$ & $\begin{array}{l}0.97 \\
(+4.3 \%)\end{array}$ & $2.61(-5.8 \%)$ & $\begin{array}{l}1.21 \\
(+9.0 \%)\end{array}$ \\
\hline
\end{tabular}

\subsection{Processing of synthetic datasets: sensitivity to noise}

As detailed in Table 3, the GV method showed low sensitivity to increasing levels of VENC-noise in the synthetic datasets: when compared to the same values extracted from the synthetic 4D-flow dataset with no added noise, WSS peak and mean values over the boundary of the analyzed sections varied by less than $5 \%$ and $3 \%$ for the TAV dataset and by less than $5 \%$ and $8 \%$ for the BAV dataset, respectively. The LP method was more sensitive to noise, although changes in WSS data remained below 10\% (Table 3).

\subsection{Analysis of the real datasets}

The WSS 3D distribution as quantified by the GV method was almost uniform in healthy volunteers, whereas high WSS spots were identified in BAV patients in the aortic arch and, to an even greater extent, in the tubular ascending aorta, where the crosssections to be further investigated were selected (Fig. 7). On these cross sections, peak WSS values as assessed via LP method were 2.90, 3.63, and 2.92 Pa in the healthy volunteers and 4.69, 5.01, and $7.59 \mathrm{~Pa}$ in the BAV patients. The position of peak WSS values was consistent with the velocity field assessed at the considered crosssections. Finally, the processing of these datasets required less than 20 minutes each: 10-15 minutes were required by manual vessel segmentation. The computation of the iso-surface, of its local normals, and of the centerline required $10.68 \pm 2.52 \mathrm{~s}$. The extraction of the 3D WSS map by the GV method and the local application of the LP method required $0.21 \pm 0.02 \mathrm{~s}$ and $1.27 \pm 0.12 \mathrm{~s}$, respectively.

\section{Discussion}

In this work, we proposed two methods exploiting the Sobel derivative filters to assess peak WSS values in the thoracic aorta from $4 \mathrm{D}$-flow datasets.

We benchmarked our methods by quantifying WSS based on synthetic 4D-flow datasets derived from the velocity field 

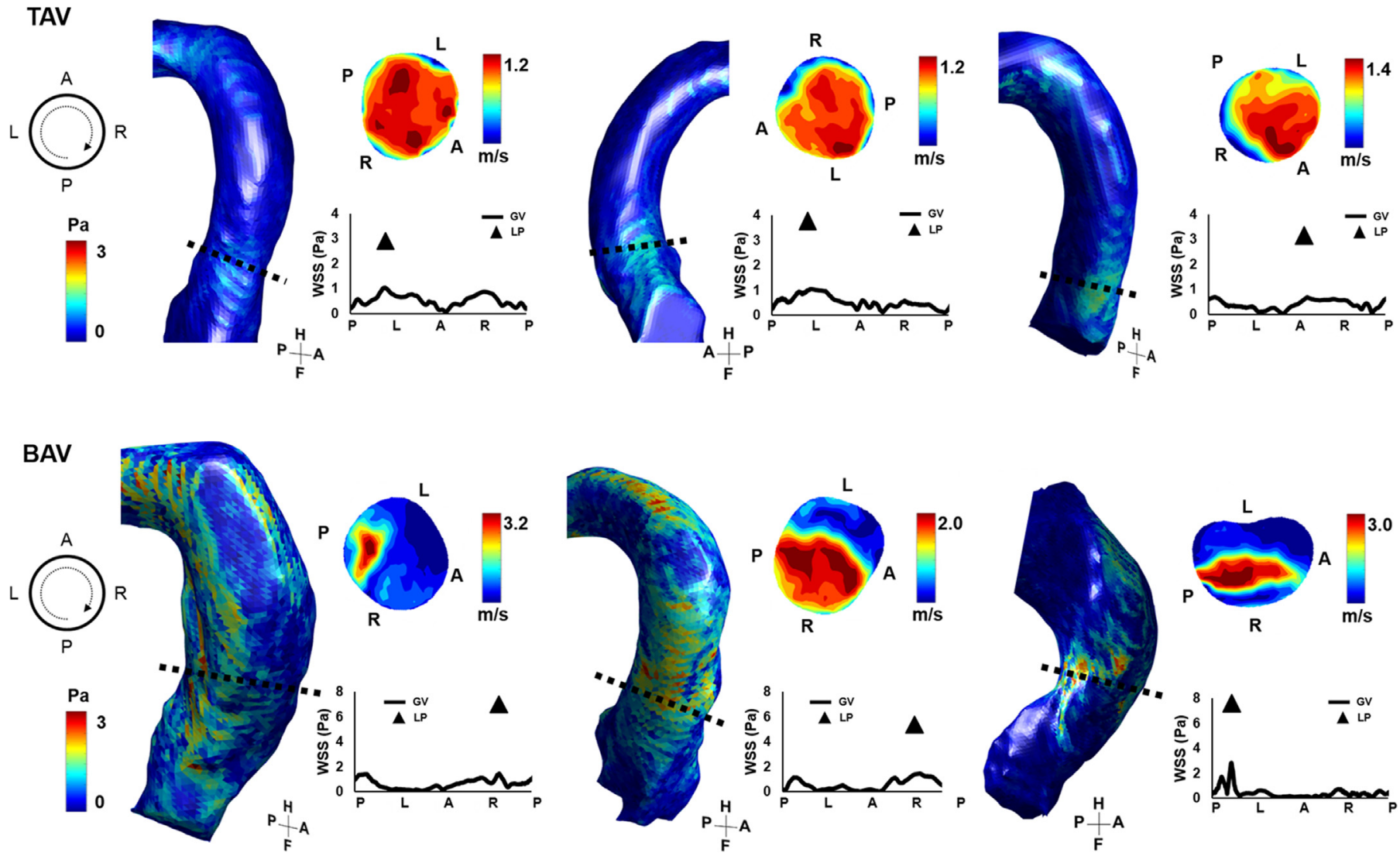

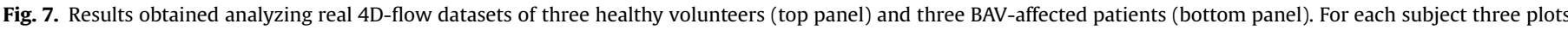

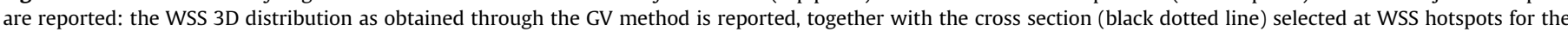

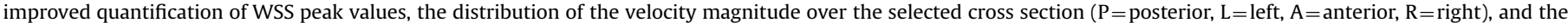
WSS distribution over the boundary of the selected cross section as yielded by the GV method, together with the WSS peak value quantified by the LP method.

computed by CFD simulations referred to healthy and BAV-affected conditions. We then used the WSS data directly computed through CFD as ground truth to assess the reliability of the two methods and their sensitivity to data spatial resolution and noise. We then applied our methods to a preliminary cohort of real datasets.

Through benchmarking, the two methods proved mutually complementary: the GV method consistently captured the ground truth WSS distribution over the aortic wall when the spatial resolution of the synthetic datasets was comparable to or better than the one suggested by current guidelines, i.e. $<2.5 \mathrm{~mm}$ (Dyverfeldt et al., 2015). To the best of our knowledge, no previous study reported WSS 3D distributions. However, the GV method underestimated peak WSS values by nearly an order of magnitude independently of the data spatial resolution. Conversely, the LP method, which is conceived to investigate WSS at user-selected cross-sections, could not yield the WSS 3D distribution. However, it notably reduced underestimation errors when computing peak WSS. In particular, owing to a quadratic dependency of WSS estimation on data spatial resolution, when the datasets with the finest spatial resolution $(1 \mathrm{~mm})$ were processed, ground truth values were underestimated approximately by a factor of 2 . Despite not being optimal, this result represents a relevant advancement as compared to currently available methods (Petersson et al., 2012). Moreover, both methods were rather insensitive to VENC-related noise (Table 3), owing to the inherent smoothing effect of the adopted 2D and 3D derivative kernels, which weight information on 6 and 18 neighbor voxels to compute spatial derivatives. Finally, and importantly, when peak WSS were located by the GV method and quantified at the located hotspot by the LP method, without exploiting any a priori knowledge on WSS yielded by CFD simulations, results remained consistent (Table 2), thus suggesting the possibility to use the two methods together in the analysis of real clinical cases.

The results of our benchmarking should be interpreted cautiously. The virtual datasets we used, although not fully idealized, were not characterized by all of the issues typically affecting real 4D-flow datasets. Even though we accounted for the effects of downsampling and of VENC-related noise, we neglected the effects of, e.g., breathing, eddy-currents, and aortic wall motion. Hence, in real world applications our approach to quantify peak WSS may be less accurate than in our benchmarking. For this reason, for the next future we plan to perform 4D-flow acquisi-tions on MRcompatible in vitro phantoms, to further test our algorithms on real datasets obtained in highly controlled and repeatable conditions.

Nonetheless, the analysis of the real datasets, although preliminary, strongly suggests that the integration of the GV and LP methods allows for correctly identifying differences in aorta peak WSS between healthy and BAV-affected subjects. We found that the average value within the latter group was $5.76 \mathrm{~Pa}, 1.83$ times higher than in the former one (3.15 Pa). This ratio is consistent with previous findings from 4D-flow analyses. In (Barker et al., 2012) peak WSS values equal to $0.4 \pm 0.3$ and $0.9 \pm 0.3$ Pa were assessed in fifteen age/size-matched controls and fifteen BAV patients. In (Bissell et al., 2013) peak WSS of $0.75 \pm 0.20 \mathrm{~Pa}$ and $1.47 \pm 0.48 \mathrm{~Pa}$ were assessed for forty-seven healthy volunteers and seventy-one stenotic BAV patients. At the same time, as compared to those peak WSS values, ours are about four times higher and better match estimations based on some highly space-and time-resolved CFD solutions, i.e., $5 \mathrm{~Pa}$ in a subject-specific 
healthy model (Lantz et al., 2011) and 8 Pa in a paradigmatic BAV model (Chandra et al., 2012), although they are still lower than the values reported by other studies, as, e.g., (Torii et al., 2013), which computed 30-40 Pa values in a patient-specific BAV model with aortic aneurysm.

In conclusion, the integration of our GV and LP methods was easy-to-implement and allowed for the time-effective quantification of peak WSS in the healthy and BAV-affected ascending aorta. Our benchmarking suggested that WSS quantification would greatly benefit from improvements in data spatial resolution, highlighting the need for technological advancements. Even if peak WSS may be quantified less reliably on real datasets than on high quality virtual ones, our preliminary analysis on real datasets of healthy and BAV-affected aortas yielded improved results as compared to previous 4D-flow based studies.

\section{Conflict of Interest}

All authors declare no conflict of interest.

\section{Acknowledgements}

This study received funding from the Italian Ministry of Health - (Young Researchers 2009 Program, Grant number GR-20091580434), the British Heart Foundation, and the H2020 EU Project AMMODIT - Approximation Methods for Molecular Modelling and Diagnosis Tools, Project ID 645672.

\section{References}

Barker,A.J.,Markl,M.,Bürk,J.,Lorenz,R.,Bock,J.,Bauer,S.,Schulz-Menger,J.,

Von Knobelsdorff-Brenkenhoff, F., 2012. Bicuspid aortic valve is associated with altered wall shear stress in the ascending aorta. Circ. Cardiovasc. Imaging 5, 457-466.

Bissell, M.M., Hess, A.T., Biasiolli, L., Glaze, S.J., Loudon, M., Pitcher, A., Davis, A., Prendergast, B., Markl, M., Barker, A.J., Neubauer, S., Myerson, S.G., 2013. Aortic dilation in bicuspid aortic valve disease: flow pattern is a major contributor and differs with valve fusion type. Circ. Cardiovasc. Imaging 6, 499-507.

Butcher, J.T., Nerem, R.M., 2006. Valvular endothelial cells regulate the phenotype of interstitial cells in co-culture: effects of steady shear stress. Tissue Eng. 12,

905-915.

Chandra, S., Rajamannan, N.M., Sucosky, P., 2012. Computational assessment of bicuspid aortic valve wall-shear stress: implications for calcific aortic valve disease. Biomech. Model. Mechanobiol. 11, 1085-1096.

Conti, C.A., Votta, E., Della Corte, A., Del Viscovo, L., Bancone, C., Cotrufo, M., Redaelli, A., 2010a. Dynamic finite element analysis of the aortic root from MRIderived parameters. Med. Eng. Phys. 32, 212-221.

Conti, C.A., Della Corte, A., Votta, E., Del Viscovo, L., Bancone, C., De Santo, L.S., Redaelli, A., 2010b. Biomechanical implications of the congenital bicuspid aortic valve: a finite element study of aortic root function from in vivo data. J. Thorac. Cardiovasc. Surg. 140, 890-896 (e2)

Della Corte, A., Body, S.C., Booher, A.M., Schaefers, H.J., Milewski, R.K., Michelena, H.I., Evangelista, A., Pibarot, P., Mathieu, P., Limongelli, G., Shekar, P.S., Aranki, S.F., Ballotta, A., Benedetto, G., Di, Sakalihasan, N., Nappi, G., Eagle, K.A., Bavaria, J.E., Frigiola, A., Sundt, T.M., 2014. Surgical treatment of bicuspid aortic valve disease: knowledge gaps and research perspectives. J. Thorac. Cardiovasc. Surg. $147,1749-1757$ (e1)

Della Corte, A., Bancone, C., Conti, C.A., Votta, E., Redaelli, A., Del Viscovo, L., Cotrufo,

M., 2012. Restricted cusp motion in right-left type of bicuspid aortic valves: a new risk marker for aortopathy. J. Thorac. Cardiovasc. Surg. 144, 360-369 (e1). Desbrun, M., Meyer, M., Schröder, P., Barr, A.H., 1999. Implicit fairing of irregular

meshes using diffusion and curvature flow. In: Proc. 26th Annu. Conf. Comput. Graph. Interact. Tech. - SIGGRAPH '99 33, pp. 317-324.

Dijkstra, E.W., 1959. A note on two problems in connexion with graphs. Numer. Math. 1, 269-271.

Dyverfeldt, P., Bissell, M., Barker, A.J., Bolger, A.F., Carlhäll, C.-J., Ebbers, T., Francios, C.J., Frydrychowicz, A., Geiger, J., Giese, D., Hope, M.D., Kilner, P.J., Kozerke, S., Myerson, S., Neubauer, S., Wieben, O., Markl, M., 2015. 4D flow cardiovascular magnetic resonance consensus statement. J. Cardiovasc. Magn. Reson. 17, 72.

El-Hamamsy, I., Chester, A.H., Yacoub, M.H., 2010. Cellular regulation of the struc ture and function of aortic valves. J. Adv. Res. 1, 5-12.

Fedak, P.W.M., Verma, S., David, T.E., Leask, R.L., Weisel, R.D., Butany, J., 2002. Clinical and pathophysiological implications of a bicuspid aortic valve. Circulation 106, 900-904.

Hope, M.D., Sigovan, M., Wrenn, S.J., Saloner, D., Dyverfeldt, P., 2014. MRI hemodynamic markers of progressive bicuspid aortic valve-related aortic disease. J. Magn. Reson. Imaging 40, 140-145.

Lantz, J., Renner, J., Karlsson, M., 2011. Wall shear stress in a subject specific human aorta - influence of fluid-structure interaction. Int. J. Appl. Mech. 03, 759-778.

Lorenz, R., Bock, J., Barker, A.J., von Knobelsdorff-Brenkenhoff, F., Wallis, W., Korvink, J.G., Bissell, M.M., Schulz-Menger, J., Markl, M., 2014. 4D flow mag-netic resonance imaging in bicuspid aortic valve disease demonstrates altered distribution of aortic blood flow helicity. Magn. Reson. Med. 71, 1542-1553.

Markl, M., Frydrychowicz, A., Kozerke, S., Hope, M., Wieben, O., 2012. 4D flow MRI. J. Magn. Reson. Imaging 36, 1015-1036.

Markl, M., Schnell, S., Barker, A.J., 2014. 4D flow imaging: current status to future clinical applications. Curr. Cardiol. Rep. 16, 481.

Michelena, H.I., Prakash, S.K., Corte, A., Della, Bissell, M.M., Anavekar, N., Mathieu, P., Bosse, Y., Limongelli, G., Bossone, E., Benson, D.W., Lancellotti, P. Isselbacher, E.M., Enriquez-Sarano, M., Sundt, T.M., Pibarot, P., Evangelista, A., Milewicz, D. M., Body, S.C., 2014. Bicuspid aortic valve identifying knowledge gaps and rising to the challenge from the international bicuspid aortic valve consortium (BAVCON). Circulation 129, 2691-2704.

Morbiducci, U., Ponzini, R., Rizzo, G., Biancolini, M.E., Iannaccone, F., Gallo, D. Redaelli, A., 2012. Synthetic dataset generation for the analysis and the evaluation of image-based hemodynamics of the human aorta. Med. Biol. Eng. Comput. 50, 145-154.

Marin, M., Palisi, M., Scognamiglio, R., Thiene, G., 1999. Aortic root dilatation in young men with normally functioning bicuspid aortic valves. Heart 82, 19-22.

Petersson, S., Dyverfeldt, P., Ebbers, T., 2012. Assessment of the accuracy of MRI wall shear stress estimation using numerical simulations. J. Magn. Reson. Imaging 36, 128-138.

Potters, W.V., Marquering, H.A., VanBavel, E., Nederveen, A.J., 2014. Measuring Wall Shear Stress Using Velocity-Encoded MRI. Curr. Cardiovasc. Imaging Rep. 7,

Roberts, W.C., Ko, J.M., 2005. Frequency by decades of unicuspid, bicuspid, and tricuspid aortic valves in adults having isolated aortic valve replacement for aortic stenosis, with or without associated aortic regurgitation. Circulation 111, $920-925$.

Shih, F.Y., 2010. Image Processing and Pattern Recognition.

Sigovan, M., Hope, M.D., Dyverfeldt, P., Saloner, D., 2011. Comparison of fourdimensional flow parameters for quantification of flow eccentricity in the ascending aorta. J. Magn. Reson. Imaging 34, 1226-1230.

Stalder, A.F., Russe, M.F., Frydrychowicz, A., Bock, J., Hennig, J., Markl, M., 2008. Quantitative 2D and 3D phase contrast MRI: optimized analysis of blood flow and vessel wall parameters. Magn. Reson. Med. 60, 1218-1231.

Torii, R., Kalantzi, M., Theodoropoulos, S., Sarathchandra, P., Xu, X.Y., Yacoub, M.H 2013. Predicting Impending Rupture of the Ascending Aorta With Bicuspid Aortic Valve: spatiotemporal Flow and Wall Shear Stress. JACC Cardiovasc. Imaging 6, 1017-1019. 